\title{
Sistemas agroforestales como alternativa de manejo sostenible en la actividad ganadera de la Orinoquia Colombiana
}

\section{Agroforestry as an alternative for sustainable management of livestock in the Orinoquia region of Colombia}

\author{
Abril YR ${ }^{1}$ \\ ${ }^{1}$ Licenciada en Producción Agropecuaria Universidad de los Llanos \\ roago2@hotmail.com
}

Recibido 14 de enero 2011 aceptado 4 de marzo 2011

\section{RESUMEN}

En los últimos años, se han acentuado esfuerzos en la búsqueda de tecnologías sostenibles, que permitan recuperar y mantener el potencial productivo de las áreas degradadas y diversificar la gama de productos, con el fin de permitir mayor competitividad. En este aspecto, es donde se ha destacado la agroforestería como alternativa sostenible para los procesos productivos del sector agropecuario. Los sistemas Agroforestales (SAF), son formas de uso y manejo de los recursos naturales en los cuales especies leñosas (árboles, arbustos, palmas) son utilizadas en asociación deliberada con cultivos o con animales en el mismo terreno, de manera simultánea o en una secuencia temporal. Dentro de las ventajas de la agroforestería se pueden mencionar la disminución de la degradación del suelo, el mejoramiento de la fertilidad del suelo con incremento en la materia orgánica y aporte de nitrógeno, control de condiciones medioambientales adversas (vientos, excesiva temperatura, evaporación del recurso hídrico, etc.), sombra y alimento para animales y disponibilidad de productos comercializables. Por tal razón, se ha postulado que los SAF más exactamente los silvopastoriles, se han combinado de diversas formas para la producción animal con árboles hacia diferentes propósitos, respondiendo en parte a los problemas de la deforestación y degradación de los ecosistemas y a la sostenibilidad de la ganadería. Los árboles fijadores de nitrógeno aparecen como 
particularmente prometedores para reducir el proceso de degradación e intensificar en forma sostenible la producción de proteína de origen animal.

Palabras clave: Suelos, zona de Colombia, degradación.

\section{ABSTRACT}

Recent years, efforts have intensified the search for sustainable technologies that can recover and maintain the productive potential of degraded areas and diversify the range of products to allow greater competitiveness. In this aspect, which has highlighted the agroforestry as a sustainable alternative to the productive processes of agriculture. Agroforestry systems (AFS), are ways of using and managing natural resources in which woody species (trees, shrubs, palms) are deliberately used in association with crops or animals in the same area, simultaneously or in a temporal sequence. Among the benefits of agroforestry include: reduced soil degradation, improved soil fertility with increased organic matter and nitrogen supply, control of adverse environmental conditions (winds, excessive temperatures, evaporation of water resources, etc.), shade and food for animals and availability of marketable products. For this reason, it has been postulated that more accurately silvopastoral SAF, have been combined in various ways for livestock with trees to different purposes, responding in part to the problems of deforestation and degradation of ecosystems and the sustainability of livestock. Nitrogen fixing trees appear as particularly promising to reduce degradation and increase sustainable in the production of animal protein.

Keywords: Soil, Colombia area, degradation.

\section{INTRODUCCIÓN}

Los sistemas tradicionales de uso de la tierra en los trópicos se han enfatizado más en la reducción de los riesgos de las cosechas que en el logro de una producción óptima. En consecuencia, los cultivos y la cría de animales, especialmente en áreas poco fértiles o propensas a la erosión, incluyen asociaciones de varias especies, cultivos intercalados y esquemas de rotación 
complejos. Muchos de esos métodos no son suficientemente productivos como para satisfacer las necesidades crecientes de la población, por ello, cada vez se hace necesario desarrollarlos y mejorarlos (Navia, 2000).

La Orinoquía colombiana ha sido tradicionalmente utilizada en sistemas de ganadería extensiva, de cría y levante, con pasturas de baja calidad nutritiva; ya que presenta limitaciones para los usos agrícolas intensivos debido principalmente a los escasos niveles de fertilidad en los suelos, pobreza en materia orgánica y elevada acidez (Galvis et al., 2008). Por tanto, los suelos de la región han sido percibidos como un recurso relativamente abundante para el sector productivo, que adquiere cada vez mayor importancia en la economía de la región (Rippstein et al., 2001).

Como un primer paso para promover el uso de nuevos enfoques de sistemas productivos agropecuarios, se requiere fortalecer la capacitación y transferencia de tecnología en manejo de SAF a productores, y de acuerdo con la experiencia se ha identificado que la metodología de diagnóstico y diseño agroforestal participativo es un procedimiento adecuado para lograrlo, (Gutiérrez y Fierro, 2006).

\section{OBJETIVOS}

Comparar y analizar los sistemas agroforestales como alternativa de manejo sostenible en la actividad ganadera de la región Orinoquia.

Conocer las principales características de cobertura y uso de los suelos, para describir los sistemas de producción ganadera que se encuentran en la región Orinoquia.

Reflexionar sobre la problemática generada por la ganadería extensiva, para poder incorporar y comprender el concepto de sistemas agroforestales.

Analizar el uso de sistemas agroforestales en los sistemas productivos ganaderos que actualmente existen en la región de la Orinoquia. 


\section{DIAGNÓSTICO DE LA ORINOQUIA}

\section{Características de la Orinoquia colombiana}

La Orinoquía colombiana, ubicada entre los 400 a $1.000 \mathrm{msnm}$, tiene una secuencia climática que va desde una gran región seca en el sector nororiental hasta un ambiente muy húmedo en el Piedemonte; es de extensa sabana que llega hasta el río Orinoco por el oriente y se extiende en sentido norte-sur desde el río Arauca hasta el río Guaviare; tiene una superficie aproximada de 266.300 km², en la cual se pueden identificar tres grandes paisajes: el piedemonte llanero, la llanura de inundación, la altillanura plana y ondulada. Otra característica importante de la Orinoquía, es la cuenca hidrográfica del río Orinoco que tiene una superficie de $347.713 \mathrm{~km}^{2}$ que corresponde al $30,4 \%$ del territorio nacional, se localiza en el nororiente del país y comprende los departamentos de Arauca, Casanare, Vichada, Meta y parte de Boyacá, Cundinamarca, Santander, Norte de Santander, Guaviare, Guainía, Vaupés, Huila, Caquetá y el Distrito Capital de Bogotá (IAVH, 2003). Los ecosistemas son generalmente frágiles, de un funcionamiento complejo que debe comprenderse mejor. Existe una rica dotación de paisajes de sabana, bosques, agua y biodiversidad (Benavides, 2008). La región tiene 13 grandes cuencas hidrográficas Figura 1; a comparación de otras regiones, la Orinoquia colombiana tiene un gran potencial hídrico para la implementación de sistemas agropecuarios.

Los suelos de la Orinoquia son de baja fertilidad, ácidos y con niveles tóxicos de aluminio y hierro; además son muy propensos a la erosión y a la degradación estructural (Rippstein et al., 2001). Las coberturas y usos predominantes de los suelos de la Orinoquia están representados en pastos, donde la vegetación de sabana, representa la mayor proporción, cuyo uso principal corresponde a ganadería extensiva y semi-intensiva (IGAC, 2002; CORPOICA, 2002) (Figura 2), un $52 \%$ de la cobertura de los suelos es de vegetación en sabanas y un $30 \%$ en tierras con pastos, reflejando que la mayoría del territorio se dedica a la producción ganadera; de ahí la importancia de mejorar sus sistemas agropecuarios, incorporando técnicas como la agroforestería. 


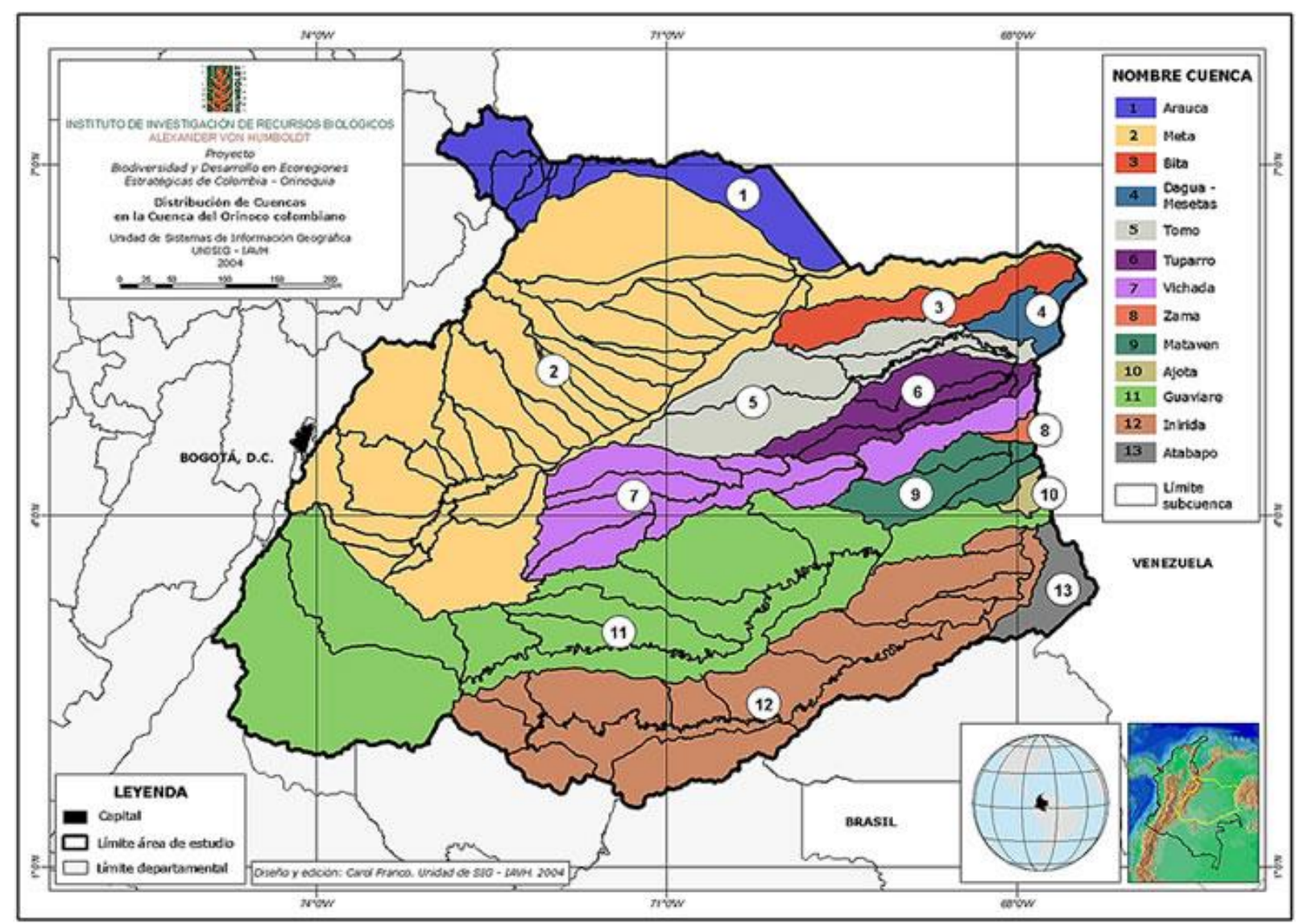

Fuente: Instituto Alexander Von Humboldt, (2004).

Figura 1. Cuencas de la Orinoquia colombiana.

La producción pecuaria en los últimos años se ha enfocado a la búsqueda de alternativas de producción en esta región, pero la ausencia de tecnologías eficientes, y la copia de modelos extranjeros no ofrecen alternativas diferentes a la explotación extensiva que se ha venido manejando (Galvis et al., 2008).

La utilización de la Orinoquía con fines agropecuarios, aunque comenzó hace más de un siglo, solamente en los últimos quince años se ha incrementado principalmente en la región del Piedemonte, enmarcada entre la cordillera Oriental, el río Metica y entre los ríos Upía y Ariari en donde existen centros urbanos importantes como Villavicencio, y por su cercanía con Santafé de Bogotá como centro importante para la comercialización de productos (Viloria, 2009). 


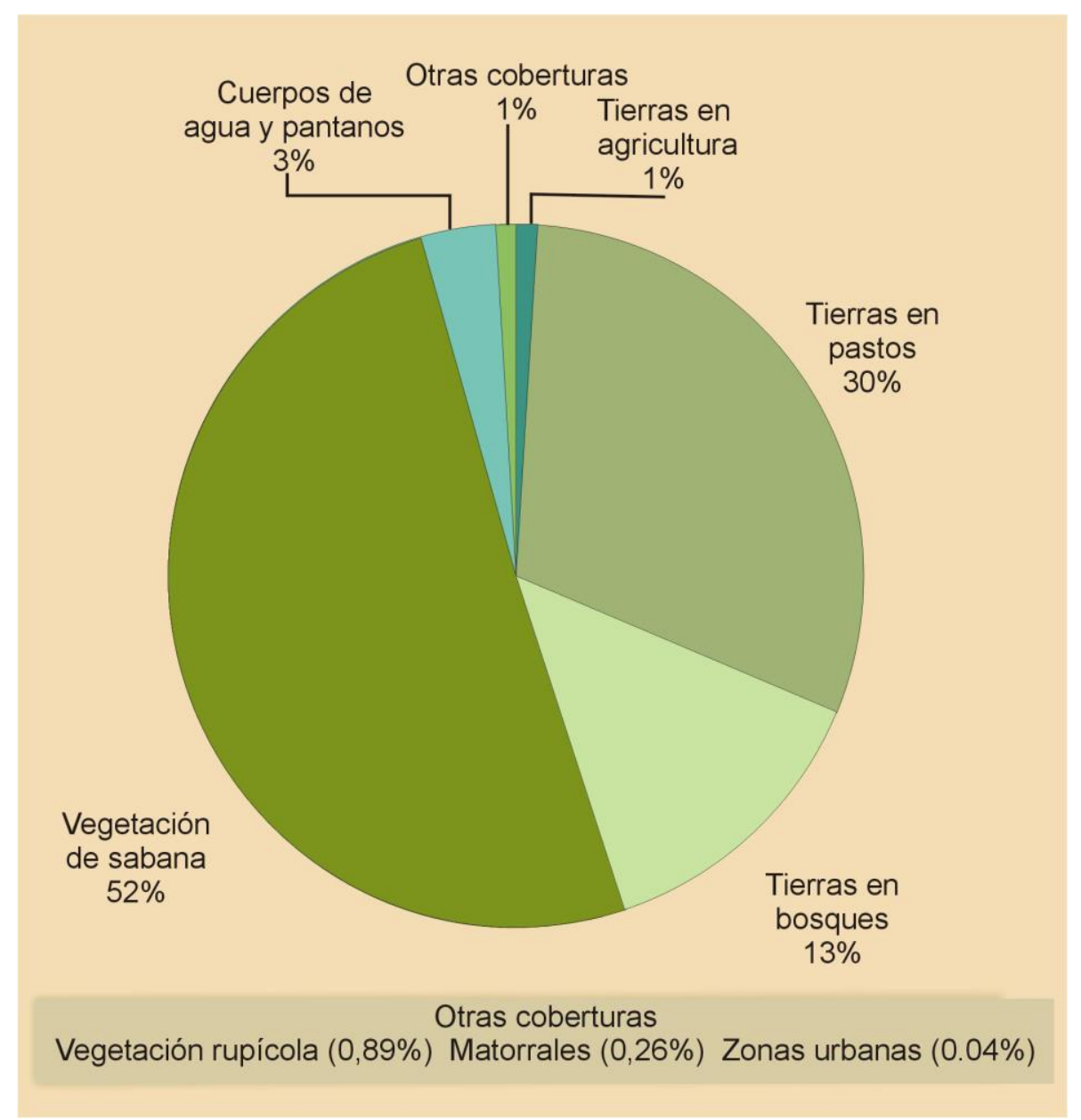

Fuente: IGAC, (2002)

Figura 2. Cobertura y Uso Actual de las tierras en la Región de la Orinoquia.

"La Orinoquia tiene el 7.2\% de las tierras agrícolas del país, de las que sólo están utilizadas 2.2\%; la mayor parte de la tierra con vocación agrícola de esta región se usa en ganadería". La ganadería se efectúa en un $90 \%$ sobre tierras con esa aptitud, mientras que las áreas cultivadas ocupan el $32 \%$ de las tierras con vocación agrícola (Rubio, 2002).

\section{Sistemas de producción ganadera desarrollados en la región Orinoquia.}

La Orinoquia es una vasta región, que tiene una baja densidad poblacional y un producto interno bruto dominado por la producción de hidrocarburos (Pinzón, 2010). La producción agropecuaria, es modesta con respecto al área regional y 
tiende al monocultivo en grandes zonas, con predilección a sistemas productivos ineficientes. La competitividad de los productos agropecuarios tradicionales es baja porque no se ha reducido el impacto de los factores geográfico y económicos, tales como la distancia, localización, baja fertilidad de los suelos, ausencia de infraestructura para gestionar la disponibilidad estacional del agua y débiles economías de multitud; algunas inversiones importantes en el agro están altamente subsidiadas y no son competitivas (Benavides, 2008).

El principio básico de la ganadería tradicional es la propiedad de un gran número de cabezas de ganado las que se cosechan una vez al año a través de los denominados trabajos del llano (trabajos en verano y la saca en junio-julio). De allí que la ganadería ocupa el primer renglón en la economía de la región, con una alta población utilizada principalmente para cría y ceba, destacándose los cruces de ganado cebú por criollo. Además se encuentran en algunas explotaciones cruces comerciales para carne, con razas como Limousin, Simmenthal y doble propósito con ganado Pardo Suizo, Normando y Holstein que se han adaptado a la región (ICA, 2008).

El sistema productivo más utilizado es la ganadería extensiva de carne, que ha sido una adaptación histórica a las condiciones de los ecosistemas regionales. En 2008, se usaron 9.75 millones de hectáreas (ha) para sostener un inventario ganadero de 5'727131 cabezas, equivalentes al $21.3 \%$ del total nacional (26'877,824 cabezas). En 2008, Meta pasó a ocupar el primer lugar de hato ganadero departamental. La explotación extensiva genera 160 mil empleos directos en la Orinoquia (un empleo por cada 61 has de pastos; Viloria, 2009). El ganado usa el $87.38 \%$ del suelo disponible en el Meta (4.68 millones de has) y el $88.97 \%$ del suelo disponible de Casanare (3.56 millones de has). La capacidad de carga en el Meta entre 2001 y 2008 fue de 1.8 cabezas/ha; en 2008, la capacidad de Casanare fue de 1.7 cabezas/ha, y la de Vichada 0.10 cabezas/ha; estas diferencias se deben principalmente, a razones de tipo tecnológico (Benavides, 2008). 


\section{PROPUESTAS}

\section{Factores que intervienen en el deterioro del suelo: "Interés en los sistemas agroforestales"}

El deterioro de la capacidad productiva del suelo se debe en gran medida a la deforestación, y surge en parte, a la demanda por el uso de la tierra; asimismo el aumento demográfico, y las presiones económicas para intensificar la producción agropecuaria, con el propósito de obtener ganancias inmediatas, son el resultado del uso inapropiado de los recursos naturales; en consecuencia, para aumentar el área de terreno disponible se incrementa la deforestación (Navia, 2000).

Es difícil estimar la velocidad con que se está efectuando la tala de selvas en las regiones tropicales, ya que los datos sobre la extensión y condiciones se hallan bastante dispersos y a menudo son imprecisos. Más de la mitad de los bosques tropicales del mundo se encuentran en América Latina (FAO, 2003). La Tabla 1 muestra la pérdida de cobertura de bosques en los principales países latinoamericanos Brasil, México, Bolivia, Perú, Venezuela y Colombia quedando en la quinta posición entre estos.

Las cifras sobre el agotamiento de bosques en Colombia demuestran categóricamente que las intervenciones relacionadas con la explotación maderera, la incorporación de nuevas tierras a la explotación agropecuaria y el avance incontrolado de obras civiles y de los cultivos ilícitos han significado la extinción del $31 \%$ de la cobertura boscosa original de la Nación, llegándose a la preocupante tasa anual de deforestación de 598.000 hectáreas (Díaz y MassCaussel, 2009). Por ende, cuando es eliminada la cubierta del bosque, los suelos se encuentran más expuestos a los efectos de las lluvias, el sol y los vientos; a ello se suma el impacto de las prácticas relacionadas con el uso de los suelos; todos estos factores hacen que las tierras sean más propensas a la erosión, y asimismo, en las zonas más lluviosas, a la compactación (Navia, 2000).

De esta manera con la erosión y la compactación, ¿Qué otros factores contribuyen a la degradación de los suelos? La utilización inadecuada de la tierra puede llevar 
a la disminución de la fertilidad, a causa de la reducción del contenido de materia orgánica y de los nutrientes; el resultado general es el deterioro de la capacidad productiva de los suelos (Rincón et al. 2008).

Tabla 1. Pérdida en la cobertura de bosques en los principales países latinoamericanos

\begin{tabular}{ccccc}
\hline País & $\begin{array}{c}\text { Superficie } \\
\text { forestal 1990 } \\
\text { (Miles de ha) }\end{array}$ & $\begin{array}{c}\text { Superficie } \\
\text { forestal 2000 } \\
\text { (Miles de ha) }\end{array}$ & $\begin{array}{c}\text { Cambio anual } \\
\text { (miles de ha) }\end{array}$ & $\begin{array}{c}\text { Tasa de } \\
\text { cambio anual } \\
\text { (\%) }\end{array}$ \\
\hline Belice & 1,704 & 1348 & -36 & $-2,3$ \\
Costa rica & 2,126 & 1968 & -16 & $-0,8$ \\
El salvador & 193 & 121 & -7 & $-4,6$ \\
Guatemala & 3387 & 2850 & -54 & $-1,7$ \\
Honduras & 5972 & 5383 & -59 & -1 \\
Jamaica & 379 & 325 & -5 & $-1,5$ \\
México & 61,511 & 55,205 & -631 & $-1,1$ \\
Nicaragua & 4,450 & 3,278 & -117 & -3 \\
Panamá & 3,395 & 2876 & -52 & $-1,6$ \\
Argentina & 37,499 & 34,648 & -285 & $-0,8$ \\
Bolivia & 54,679 & 53,068 & -161 & $-0,3$ \\
Brasil & 566,998 & 543,905 & $-2,309$ & $-0,4$ \\
Colombia & 51,06 & 49,601 & -190 & $-0,4$ \\
Ecuador & 11,929 & 10,557 & -137 & $-1,2$ \\
Guyana & 17,365 & 16879 & -49 & $-0,3$ \\
Paraguay & 24,602 & 23,372 & -123 & $-0,5$ \\
Perú & 67,903 & 65,215 & -269 & $-0,4$ \\
Venezuela & 51,681 & 49,506 & -218 & $-0,4$ \\
\hline
\end{tabular}

Fuente: FAO, (2003).

En efecto, la tala excesiva amenaza el bosque de muchas maneras, y daña así, el bienestar social y económico de los seres humanos. Uno de los efectos de la deforestación es la pérdida de biodiversidad, así como la degradación del suelo que es la disminución de su capacidad para soportar vida, no solo la vegetal, que es la más aparente, sino también la de la microflora y de la fauna propia del mismo; se dice que la mayoría de los estudios coinciden que la principal causa de la deforestación de América Latina es la creación y manejo inadecuado de fincas ganaderas. De allí el gran reto de la ganadería tropical moderna que consiste en 
aumentar la producción de carne y leche en forma acelerada y sostenida para suplir las demandas de una población creciente, además, de garantizar la conservación de los recursos naturales y el medio ambiente (Sotomayor, 2004).

Se han propuesto los SAF como una alternativa adecuada para aumentar y mantener la producción agropecuaria a largo plazo, que varían de acuerdo al estado de los terrenos (Sotomayor, 2004). Los SAF pueden contribuir a solucionar problemas de uso de los recursos naturales debido a las funciones biológicas y socioeconómicas, al obtener productos como madera, leña, frutas, forraje y postes (Vargas y Sotomayor, 2004).

\section{Conceptos básicos de sistemas agroforestales}

A través de los años se han dado numerosas definiciones del término "agroforestería", algunas de las cuales suponen condiciones muy específicas y otras suponen la existencia de áreas superpuestas; entre otras: los SAF son formas de uso y manejo de los recursos naturales, donde las especies leñosas (árboles, arbustos, palmas) son utilizadas en asociación deliberada con cultivos agrícolas o con animales en el mismo terreno, de manera simultánea o en una secuencia temporal. Lo cual está de acuerdo con lo que expone (Meza, 2003), quien afirma que los SAF son la combinación de los sistemas tradicionales de producción agrícola y ganadero con el forestal; practicados en la misma unidad de tierra, alternada o simultáneamente con el fin de proveer estabilidad ecológica y beneficios sostenibles a los productores. Como ejemplos de SAF pueden mencionarse los cultivos perennes (café y cacao) bajo sombra de árboles, cultivos anuales intercalados con plantaciones, huertos caseros mixtos, combinaciones de árboles con pastos, plantas para forraje, cultivos en franjas, cercas vivas, cortinas rompevientos y algunas formas de la agricultura migratoria (Montagnini, 2000). La Figura 3, el diagrama del sistema agroforestal muestra todas las interacciones entre los diferentes componentes, teniendo en cuenta las condiciones ambientales, los insumos y la administración como mano de obra utilizada para su adecuado funcionamiento. 
La agroforestería es una interdisciplina, también una tradición e innovación productiva y de conservación de la naturaleza, donde existen formas de manejo y aprovechamiento de SAF en fincas y territorios comunitarios para obtener una producción libre de agroquímicos y duradera, con predominio y desarrollo de saberes tradicionales y novedosos, fortaleciendo la identidad cultural, interacciones ecológicas totales de complementariedad del sistema, diversificación del paisaje, aprovechamiento adecuado de recursos naturales, privilegio del trabajo humano, uso de tecnologías de bajo impacto ambiental y relaciones sociales y económicas de bienestar, equidad y justicia (Ospina, 2003).

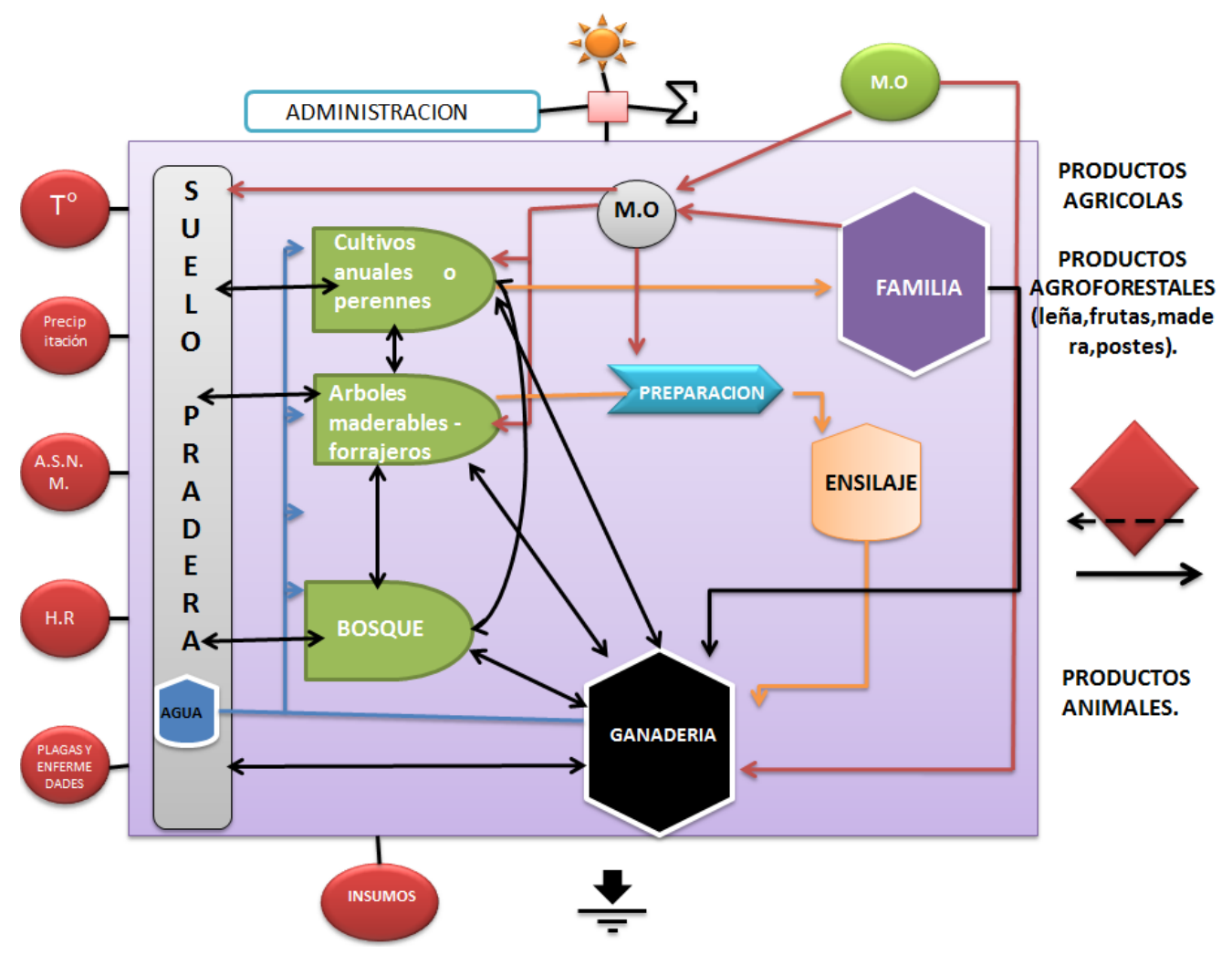

Fuente: Adaptado de Montagnini, (2000).

Figura 3. Diagrama de un sistema agroforestal.

Debido a la gama de tendencias en las definiciones, pueden asignarse varias categorías o descripciones de aspectos a los cuales hace referencia: Ospina, (2004) (Tabla 2). 
Tabla 2. Categorías que se registran en las definiciones de la agroforestería

\begin{tabular}{cl}
\hline DESCRIPTOR & \multicolumn{1}{c}{ DEFINICIÓN } \\
\hline Carácter & $\begin{array}{l}\text { Hace referencia a la práctica y estudio de la agroforestería. } \\
\text { Tipo de componentes (o productos) y distribución } \\
\text { espaciotemporal en el área o unidad de tierra. }\end{array}$ \\
Productividad & $\begin{array}{l}\text { Mantenimiento o aumento de ella, en comparación con } \\
\text { monocultivo, monoplantación, y monocrianza. }\end{array}$ \\
Conservación/s & $\begin{array}{l}\text { Mantenimiento de la producción y conservación de recursos } \\
\text { naturales, en algunas ocasiones figuran aspectos } \\
\text { socioeconómicos asociados. }\end{array}$ \\
Interacciones & $\begin{array}{l}\text { Existencia de interacciones biológicas/ecológicas entre } \\
\text { componentes agroforestales, independientemente de su } \\
\text { magnitud. }\end{array}$ \\
Socioculturales & $\begin{array}{l}\text { Origen y práctica de la agroforestería, beneficio de } \\
\text { comunidades locales o sociedad. }\end{array}$ \\
\hline
\end{tabular}

\section{Tipos de Sistemas Agroforestales}

Como parte de la búsqueda de soluciones a los problemas ambientales, las instituciones de investigación han comenzado la tarea de sistematizar, clasificar y enriquecer todo conjunto de prácticas y sistemas de manejo mixto que utilizan a la vez árboles, cultivos y ganado; como estrategia apropiada de explotación que supera el manejo tradicional de los sistemas agropecuarios que se han venido manejando. Este nuevo enfoque de producción se ha agrupado bajo el término de Modelos agroforestales (Gutiérrez y Fierro, 2006). La Figura 4 muestra la interacción de los componentes agrícola - pecuario - bosques, dando como resultado cada uno de los tipos de SAF de acuerdo a su combinación.

Las definiciones de cada uno de los Modelos Agroforestales se basan según Meza (2003):

Agrosilvopastoriles: son sistemas donde se combinan agricultura, árboles y ganadería, aprovechando los espacios con cultivos, para después cosecharlos, y sembrar praderas con uso de pastoreo rotacional.

Silvopastoriles: SAF donde se combinan los bosques, praderas y animales domésticos. Su importancia radica en que se presenta una mayor producción de 
biomasa masticable en comparación con la que se obtiene con monocultivo de pastos; además, de la integración de la producción agrícola y ganadera se racionaliza el uso de los recursos, y se obtiene autosuficiencia.

Agrosilvoculturales: en estos sistemas se combinan árboles y/o arbustos con cultivos; En este caso se pueden asociar en forma de callejones o entre las hileras.

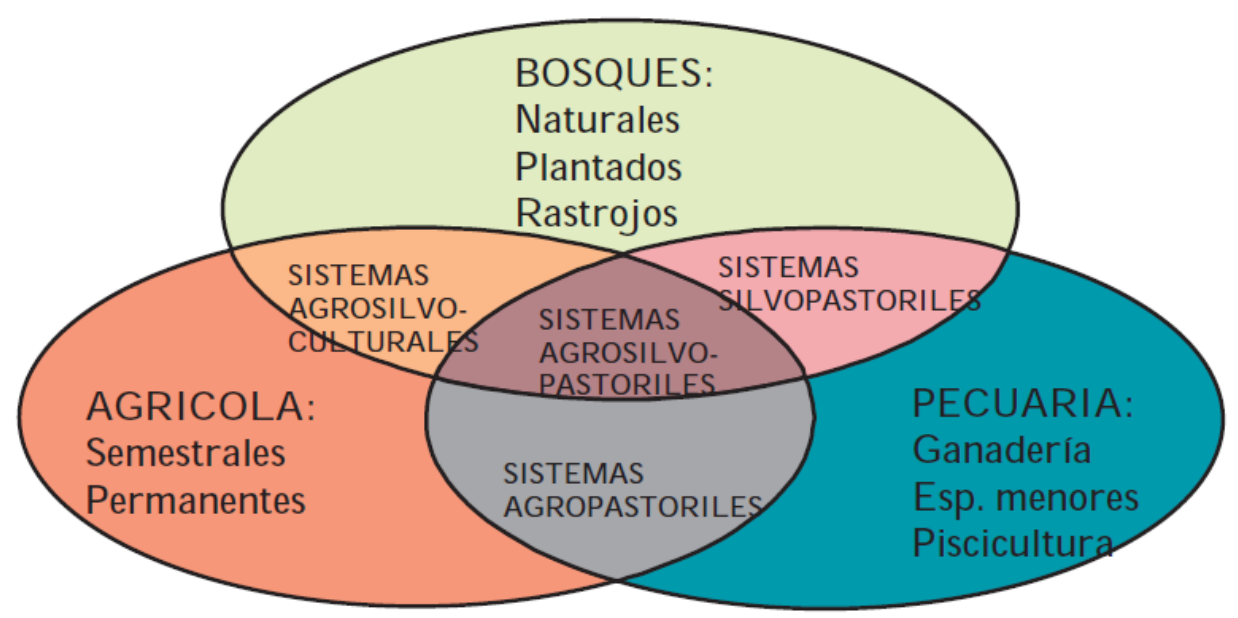

Fuente: Cipagauta y Orjuela (2003).

Figura 4. Modelos Agroforestales.

\section{Sistemas agroforestales asociados a la ganadería}

La actividad ganadera genera el $3 \%$ de la riqueza nacional, participa con el $26 \%$ del PIB agropecuario y representa el $60 \%$ del PIB pecuario. La ganadería además, es la actividad económica con mayor presencia efectiva en el difícil papel de ocupación pacífica del territorio rural. En muchas regiones, es toda una cultura que pertenece al quehacer cotidiano de sus pobladores (Murgueitio et al., 2008).

Sin embargo, la ganadería puede incrementar significativamente sus contribuciones económicas y sociales; los sistemas silvopastoriles (SSP) son fundamentales para el proceso de cambio. Estos pueden tener, además de la actividad pecuaria, otros usos complementarios como son la producción de madera y frutos; el beneficio de un microclima más benigno, la oferta de hábitat 
para la fauna silvestre, la regulación hídrica en cuencas hidrográficas y una mayor belleza del paisaje (Murgueitio et al., 2008).

Entonces se dice que los SSP, son una práctica agroforestal que combina árboles o arbustos con praderas y ganado, con el objetivo de obtener productos forestales madereros (madera, postes, leña, carbón) y (frutos, miel, hongos y otros), productos derivados del ganado (carne, lana, leche y otros), y forraje de la pradera (Ojeda y Restrepo, 2003). La Figura 5 muestra el esquema del sistema silvopastoril en el que se determina las diferentes relaciones reciprocas que se presentan entre los componentes en un mismo terreno.

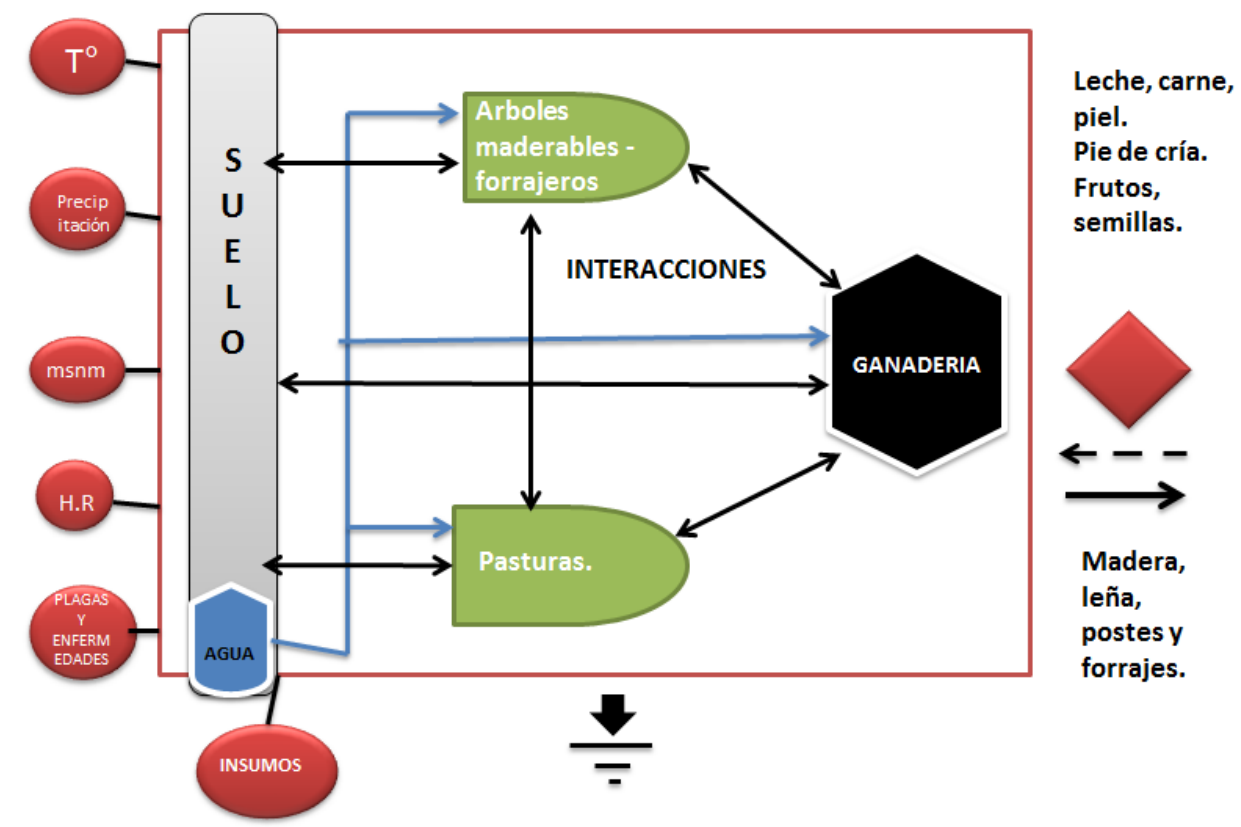

Fuente: Adaptado de Ojeda y Restrepo, 2003.

Figura 5. Diagrama de un sistema silvopastoril

Según Ojeda y Restrepo, (2003) los SSP se pueden clasificar en:

- Los árboles y arbustos dispersos en potreros: donde la vegetación está constituida por la combinación de árboles y/o arbustos con pastos dispersos uniformemente en el terreno.

- Especies arbóreas en callejones: consiste en establecer los árboles y/o arbustos en una o más hileras en la pradera. 
- Cercas vivas: consiste en utilizar las especies arbóreas como cerco, ya sea en una o más hileras.

- Barreras rompevientos: son utilizadas en suelos con pendientes, para otorgar protección al suelo, disminuir escurrimiento superficial y evitar su pérdida, y sectorizar áreas de producción, donde se protege de los vientos a cultivos y animales.

Las ventajas de los SSP para el productor ganadero es que se produce forraje, ya sea en forma de ramoneo o de corte, se mejoran las condiciones del suelo por el aporte de materia orgánica, aumenta la diversidad de especies y de insectos benéficos que ayudan a controlar los daños ocasionados por plagas y enfermedades; al mismo tiempo protegen al ganado de las altas temperaturas y les dan mejores condiciones fisiológicas y por ultimo diversifican la producción en la finca y disminuyen la utilización de insumos externos (Cipagauta y Andrade, 2001).

\section{Sistemas Agroforestales existentes en la Orinoquia}

Implementar los SAF, es resolver un problema tecnológico de un sistema de producción regional; ejemplo CORPOICA ha trabajado en SAF con caucho en la altillanura "para hacer suelo", con plátano buscando reducir la severidad de la sigatoka negra, con ganadería resolver el problema de la fisiología del animal y de nutrición (Gutiérrez y Fierro, 2006). Los tipos de SSP que se observan en la Orinoquia son: cercas vivas (Fotografía 1), árboles dispersos (Fotografía 2), callejones (Fotografía 3) y en plantaciones (Fotografía 4).

\section{DISCUSIÓN}

La degradación de las praderas, la falta de pastos mejorados, la baja carga animal, el manejo de la sabana nativa entre otras, no resultan siendo problemas tan importantes como la falta de atención a observar y evaluar los procesos del sistema ganadero por parte de sus dueños; hace falta a la actividad pecuaria darle un enfoque más tecnológico con capacitación, y dejar de considerar y entregar el 
manejo de los hatos a la limitada capacidad profesional realizada por los vaqueros, sin menospreciar el conocimiento empírico que estas personas tienen.

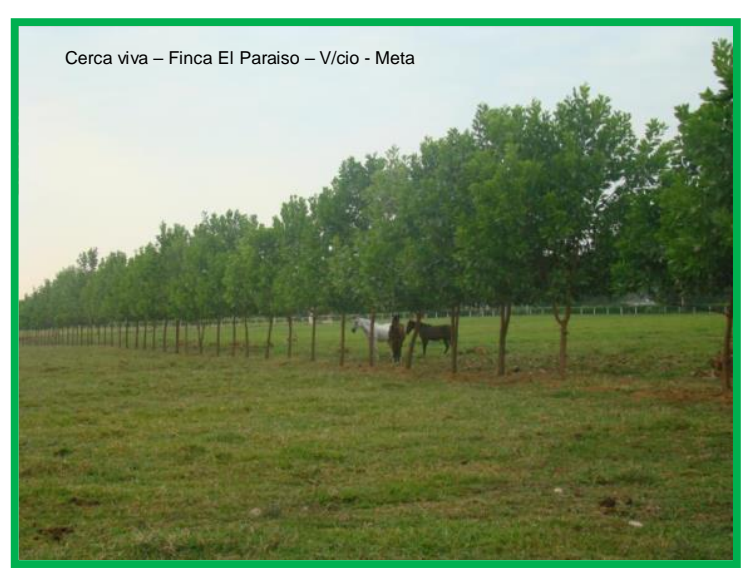

Fuente: Martínez, CORPOICA

Fotografía 1. Cerca viva, VillavicencioMeta.

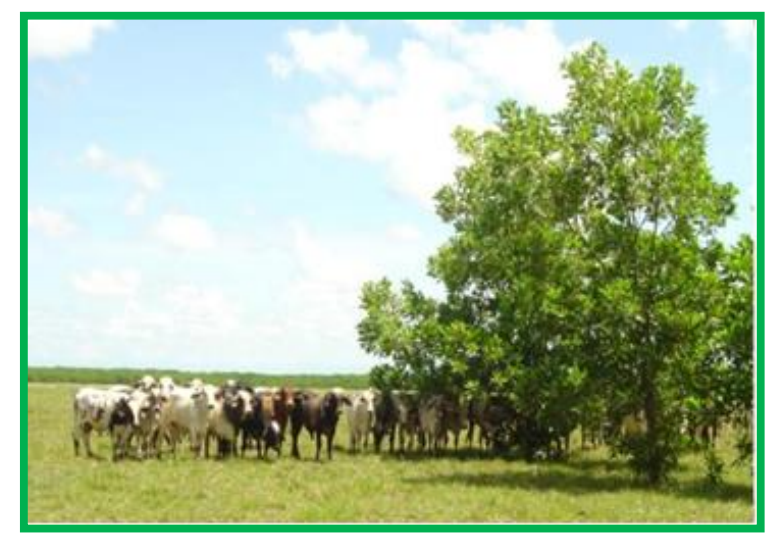

Fuente: Martínez, CORPOICA.

Fotografía 3. Acacia mangium en franjas de pasturas en Puerto GaitánMeta.

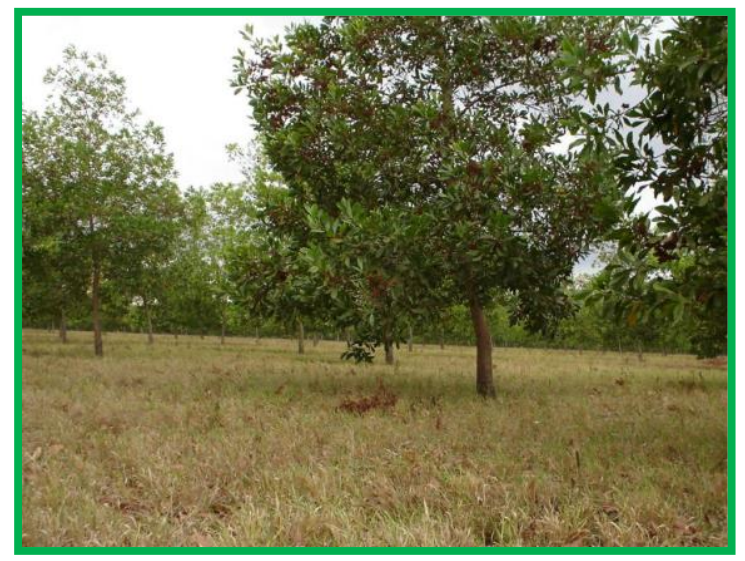

Fuente: Martínez, CORPOICA.

Fotografía 2. Sistema silvopastoril con Acacia mangium, Puerto. López-Meta.

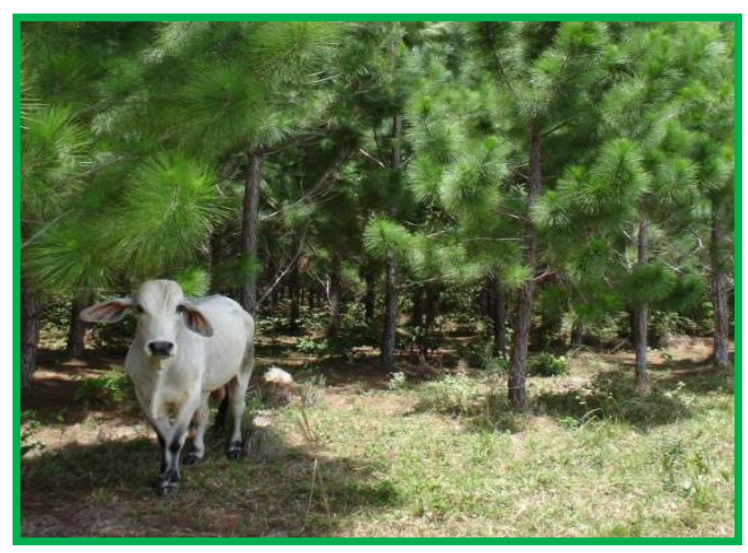

Fuente: Martínez, CORPOICA.

Fotografía 4. SAF con Pino caribaea, Villanueva - Casanare.

Son pocos los ganaderos de la región que llevan registros de sus explotaciones, donde se pueda analizar productos y ganancias económicas principalmente. Es el caso, de que al querer recopilar datos e información para el estudio de los sistemas de producción no es posible, por esta razón la actividad ganadera conduce a un bajo rendimiento, trayendo como consecuencia un balance negativo en la sostenibilidad de los hatos. 
El grado de erosión, desertificación, pérdida de agua y fertilidad del suelo, son consecuencias ocasionadas por el mal uso y manejo de los sistemas agropecuarios, la Tabla 3 muestra que la erosión es una de las problemáticas ambientales más relevantes en el país, por el inútil aprovechamiento del suelo; por tanto es significativo en un futuro trabajar para conseguir un aprovechamiento racional del recurso, con políticas de extensión, fomento e investigación, que atienda las necesidades del país en materia de conservación y recuperación del agua y suelo.

Tabla 3. Antecedentes - Situación Nacional, del territorio nacional (1’141.748 $\mathrm{km}^{2}$ )

\begin{tabular}{ccc}
\hline Afectado por erosión & Desértico & Presenta déficit d agua. \\
\hline $35 \%$ & $4.3 \%$ & $14 \%$ \\
\hline
\end{tabular}

Fuente: IGAC, CORPOICA, (2002)

Reiterando lo anterior, la restauración de suelos sobre utilizados o erosionados requiere cambios importantes en las prácticas agrícolas y pecuarias, investigadores del CIAT, advierten que "bajo condiciones naturales, los suelos de sabana del trópico colombiano, no ofrecen un medio apropiado para el desarrollo agrícola sostenible".

Se tiene que empezar por hacer un diagnóstico participativo del sistema agropecuario con ayuda de profesionales en las fincas, antes de reestructurar el sistema productivo, evitando invertir en cambios que tal vez no sean necesarios, posiblemente con este diagnóstico se pueda rescatar manejos importantes del sistema; y así la implementación de tecnologías sea una manera de extensión agropecuaria que se pueda llevar a cabo sin imponer cambios tan bruscos en los sistemas productivos ganaderos y en la cultura regional que se tiene. Para esto es importante clasificar las actividades agropecuarias más sobresalientes de la región Orinoquia, las cuales son la ganadería, el cultivo de arroz, y actualmente el incremento de la palma de aceite; según estadísticas realizadas por Viloria (2009) (Finagro, 2010a; 2010b) (Tabla 4). 
Tabla 4. Los tres productos agropecuarios más importantes de la Orinoquia

\begin{tabular}{|c|c|c|c|c|c|c|}
\hline Ganadería & Meta & Casanare & Arauca & Vichada & Colombia & Producto \\
\hline \multirow{6}{*}{ Arroz } & Has.Pastos & $4,682,016$ & $3,557,129$ & $1,512,443$ & N.D & $39,152,358$ \\
\hline & Cabezas & $2,656,570$ & $2,135,561$ & 800,000 & $\begin{array}{c}135,000 \\
\text { apr. }\end{array}$ & $26,877,824$ \\
\hline & & $\begin{array}{l}\text { Riego } \\
26649\end{array}$ & $\begin{array}{l}\text { Riego } \\
25067\end{array}$ & Secano & Secano & $\begin{array}{c}\text { Riego } \\
256,643\end{array}$ \\
\hline & $\mathrm{Ha}$ & $\begin{array}{l}\text { Secano } \\
\text { Mec. } \\
46,100\end{array}$ & $\begin{array}{c}\text { Secano } \\
\text { Mec } \\
34,774\end{array}$ & $\begin{array}{l}\text { Mec. } \\
3,595\end{array}$ & $\begin{array}{l}\text { Mac. } \\
22\end{array}$ & $\begin{array}{c}\text { Secano mec. } \\
143,533 \\
\text { Secano mac. } \\
60,591\end{array}$ \\
\hline & & Riego & Riego & & & $\begin{array}{c}\text { Riego } \\
1,714,908\end{array}$ \\
\hline & Toneladas & $\begin{array}{c}150,359 \\
\text { Secano } \\
\text { Mec. } \\
259,842\end{array}$ & $\begin{array}{c}145,151 \\
\text { Secano } \\
\text { Mec. } \\
181,959\end{array}$ & $\begin{array}{c}\text { Secano } \\
\text { Mec. } \\
13,989\end{array}$ & $\begin{array}{l}\text { Secano } \\
\text { Man. } \\
30\end{array}$ & $\begin{array}{c}\text { Mec. } \\
687,324 \\
\text { Secano } \\
\text { Mac. } \\
90,880\end{array}$ \\
\hline \multirow[t]{2}{*}{$\begin{array}{l}\text { Palma de } \\
\text { aceite }\end{array}$} & $\mathrm{Ha}$ & 83,000 & 11,870 & N.D & N.D & 262,742 \\
\hline & Toneladas & 222,000 & 53,319 & N.D & N.D & 914,761 \\
\hline
\end{tabular}

Fuente: Viloria, (2009); Finagro, (2010a; 2010b).

Con un $31.8 \%$ la región de la Orinoquia cuenta con un gran potencial para la implementación de los SAF, superando a regiones como la Andina y el Caribe que se destacan por su experimentación, investigación, estudio, y desarrollo en estas técnicas (Figura 6); lo paradójico es que la región Orinoquia tiene alternativas para mejorar los sistemas agrícolas y pecuarios, ya que cuenta con dos factores importantes, que son su extensión en tierras y su actividad económica principal, que es el sector agropecuario, los cuales no está aprovechando. 


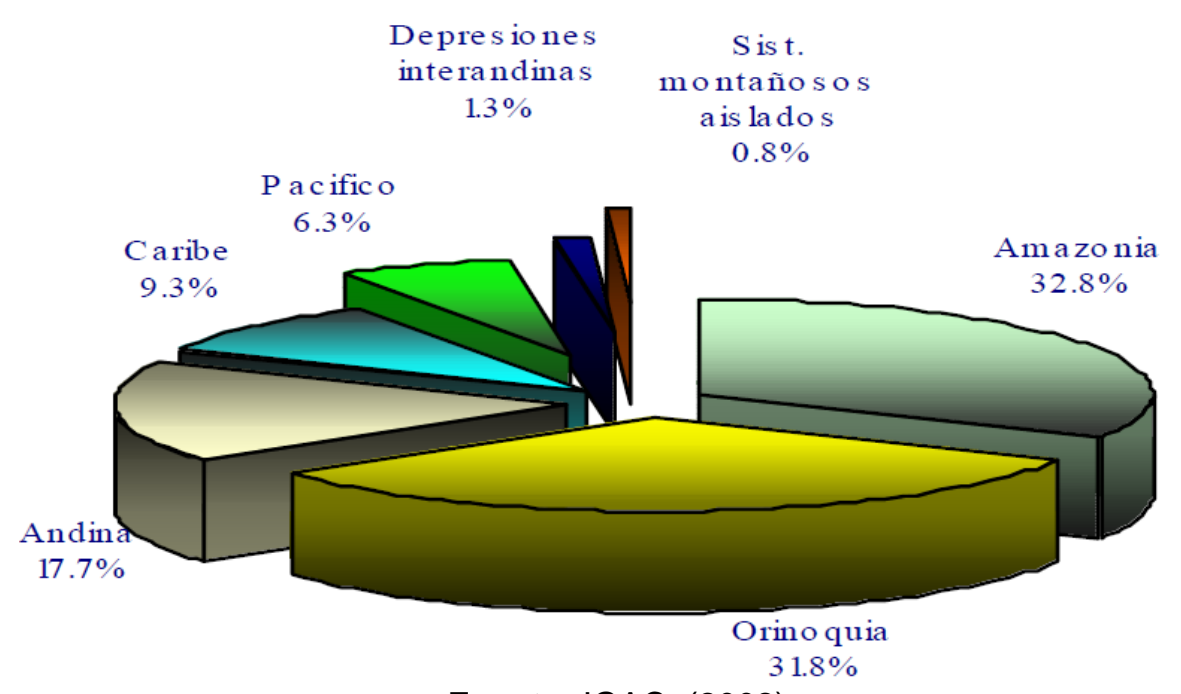

Fuente: IGAC, (2002)

Figura 6. Usos potenciales Agroforestales por regiones naturales

La estrategia de construcción de capa arable propuesta por los SAF es central, para cambiar las actuales proporciones de uso de los suelos de la Orinoquia, los cuales apuntan a cultivos de biocombustibles y ganadería extensiva; práctica convenida por los gobiernos locales que no son ajustados a los sistemas productivos de la región. En contraste con estas prácticas, varios investigadores registran experiencias positivas en la introducción de métodos agrícolas y pecuarios integrados. Ejemplo de esto lo busca y orienta el SSP, que relaciona la ganadería con los cultivos de principal actividad en la región (Figura 7).

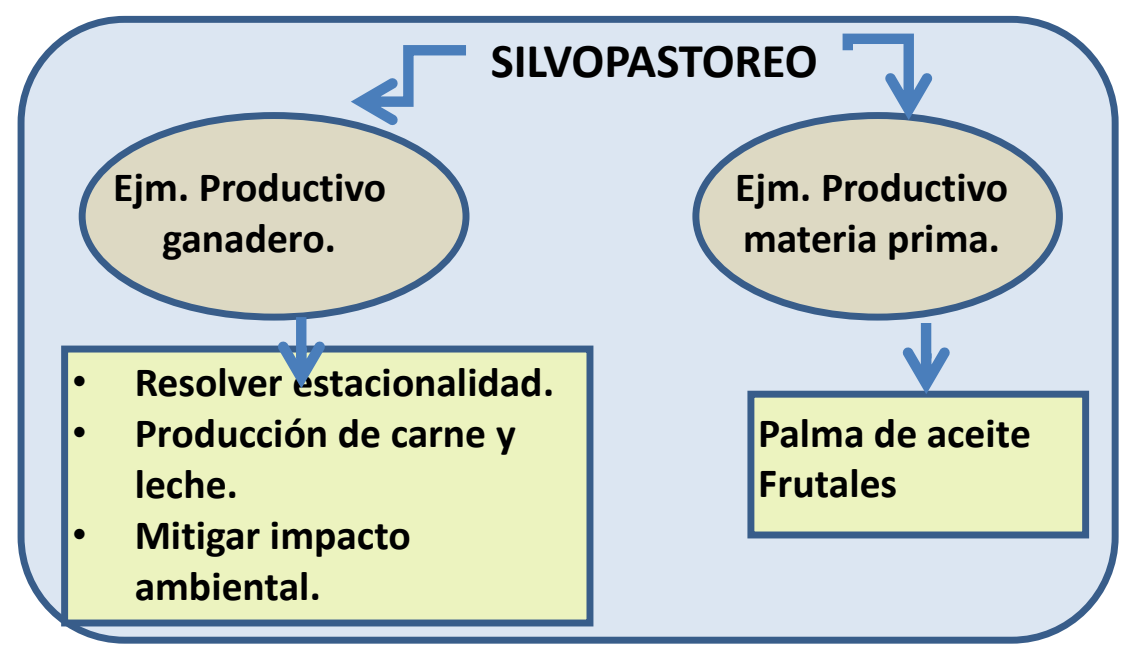

Fuente: IGAC, (2002)

Figura 7. Orientación de los sistemas silvopastoriles. 
Es muy importante mencionar, que cuando hay mayor cantidad de componentes en un sistema, las pérdidas de energía se reducen, lo cual se logra cuando en una finca, se integran sus subsistemas, por ejemplo, las praderas, los animales, los árboles plantados, los bosques nativos, la fauna y flora silvestre, los nacimientos de agua, y otros factores en un solo SSP. Al comparar las dos formas de cobertura de áreas con SSP; indica que el 68\% a nivel nacional, prefiere las asociaciones de todos los elementos que interactúan en el sistema productivo; reiterando que la actividad ganadera con SSP domina sobre los tipos de SAF a implementar en el país, a diferencia del uso agrícola que muestra el 33\% del territorio (Tabla 5).

Tabla 5. Composición de la cobertura de las áreas en silvopastoreo

\begin{tabular}{lccc}
\hline \multicolumn{1}{c}{ Uso predominante } & Área (ha) & $\begin{array}{c}\% \text { Agrofor. } \\
\text { Nal. }\end{array}$ & $\begin{array}{c}\% \text { Nal Pastos o } \\
\text { Uso agrícola }\end{array}$ \\
\hline $\begin{array}{l}\text { Pastos naturales con árboles y } \\
\text { arbustos }\end{array}$ & 3.001 .155 & $14.9 \%$ & $7.2 \%$ \\
$\begin{array}{l}\text { Asociación de pastos, rastrojos, } \\
\text { relictos de bosque. Pastoreo }\end{array}$ & 13.767 .786 & $68 \%$ & $33.0 \%$ \\
extensivo madera, fibra y fruto uso \\
doméstico.
\end{tabular}

Fuente: IGAC, (2002).

La importancia de lo consultado y expuesto sobre los SAF asociados a la ganadería, es información que conjuntamente llevan a cabo instituciones de investigación, los cuales expresan resultados de la implementación de SAF en diferentes contextos; pero cuando se quiere reunir y conocer datos para hacer un diagnóstico de la región, se halla que hay poca información acerca de investigaciones e implementación de dichas técnicas, y muchas de las experiencias no están aún publicadas. Por lo que es indispensable realizar más indagación, ensayos y estudios sobre la ejecución de SAF en la región Orinoquia, teniendo en cuenta que es una de las regiones con más potencial en dichas tecnologías. 


\section{CONCLUSIONES}

- Los sistemas de producción asociados a agroforestales presentan grandes expectativas entre los productores y las instituciones de investigación, como una alternativa de uso de la tierra, por los beneficios agregados que generan estas tecnologías en lo social, en los servicios ecológicos y en las funciones productivas, como consecuencia del manejo e interacción entre árboles, suelo, pastos y animales.

- Con un 52\% la cobertura de los suelos en la región Orinoquia es de vegetación en sabanas y un $30 \%$ en tierras con pastos, reflejando que la mayoría del territorio se dedica a la producción ganadera; de ahí la importancia de mejorar y realizar adecuados diagnósticos para la implementación de SAF asociados a los sistemas de producción más significativos en la economía de la región.

- Los antecedentes de la situación nacional, indican que el grado de erosión es una de las problemáticas más sobresalientes, con un 35\% del territorio afectado, seguido por el $14 \%$ en pérdida de agua, y un $4.3 \%$ en desertificación de los suelos; consecuencias ocasionadas por el mal uso y manejo de los sistemas agropecuarios.

- La pérdida de cobertura de bosques en los principales países latinoamericanos los representa Brasil, México, Bolivia, Perú, Venezuela y Colombia quedando en la quinta posición, con la extinción del $31 \%$ de la cubierta boscosa original del país, llegándose a la preocupante tasa anual de deforestación de 598.000 hectáreas

- Como un primer paso para promover el uso de los SAF, se requiere fortalecer la capacitación y transferencia de tecnologías a productores. Al mismo tiempo que las políticas gubernamentales, sean consolidadas y de mejor representación, para que el débil sector agropecuario reciba más apoyo del estado. 
- Los SSP contribuyen al incremento de la fertilidad del suelo, reciclaje y conservación de nutrientes a través de la diversificación de la cobertura vegetal, disminuyendo la presión sobre los relictos de bosque todavía presentes en las zonas de producción, por lo tanto, las leguminosas arbóreas en los sistemas ganaderos sin lugar a dudas ocupan un papel fundamental en la relación suelo-planta-animal, mejorando así la eficiencia de utilización de nutrientes y la respuesta animal.

- Los SSP incrementan la producción de forrajes y alimentos de alta calidad nutritiva, particularmente en la época de verano; igualmente permite elevar la oferta de productos del sistema productivo para el auto consumo o el mercado.

- Por todo lo anteriormente planteado, por cada día que pasa y no se siembran arboles multipropósito en las fincas, es un tiempo precioso que nunca se recupera y siempre se dirá porque no se hizo antes, es el momento de devolverle los árboles a la ganadería y los beneficios serán notorios.

- Al comparar que la relación de los SAF con el sistema de producción ganadera son una cadena de beneficio, se demuestra que sin uno no está el otro, dado que la falta de árboles significa falta de protección del suelo, pérdida de suelo, representa perdida de bosque y de agua, y esto simboliza la disminución de la producción agropecuaria, y por último la pobreza. Por eso Los SAF son una alternativa de uso de los recursos naturales que disminuye el daño ocasionado por los sistemas tradicionales.

- La iniciativa de implementar los SSP en la Orinoquia busca demostrar que es posible lograr cambios en las formas y métodos de producción convencionales de las fincas ganaderas que causan impactos negativos, si se compensa a los productores por los servicios ambientales que generan a través de estos cambios tecnológicos, ofreciendo además la asistencia técnica que es tan importante para todo sistema productivo. 
- Pero es notorio que la región Orinoquia no ha aprovechado el $31.8 \%$ de potencial que se tiene para ejecutar los SAF, favorecido por su extensión en tierras y su cultura en sistemas productivos agropecuarios; por consiguiente se necesita realizar más investigación, experimentación y estudios sobre la implementación de tecnologías que mejoren los procesos agrícolas y pecuarios de la región.

\section{REFERENCIAS BIBLIOGRÁFICAS}

1. Benavides, J. El desarrollo económico de la Orinoquia como aprendizaje y construcción de instituciones. Facultad de administración de la universidad de los andes. 2008.

2. Benavides J. "Cuatro micro-ensayos sobre infraestructura y geografía en Colombia." Working Paper. Facultad de Administración de la Universidad de los Andes. 2008.

3. Cipagauta M, Andrade H. sistemas silvopastoriles. Corpoica, 2001.

4. Cipagauta M, Orjuela J. Utilización de sistemas agrosilvopastopriles para contribuir a optimizar el uso de la tierra en el área intervenida de la amazonia. Corpoica, 58 p. 2003.

5. Díaz J. Mas-Caussel J. Fs. La deforestación de los bosques tropicales: una revisión Instituto de Geografía, Universidad Nacional Autónoma de México. Unidad Académica Morelia, Morelia Michoacán, México. 2009.

6. FAO. Situación de los bosques del mundo 2003. Roma. ftp.fao.org/docrep/fao/005/y7581s/y7581s00.pdf. 2003.

7. FINAGRO. Arroz. Sistema de información sectorial: http://www.FINAGRO.com.co/html/cache/gallery/GC-8/G-11/arroz.pdf. 2010a.

8. FINAGRO. Palma. Sistema de información sectorial. Recuperado el 10 de enero de 2010 en: http://www.FINAGRO.com.co/html/cache/gallery/GC-8/G11/palma.pdf. 2010b.

9. Galvis J, Amézquita E, Madero E. Evaluación del efecto de la intensidad de labranza en la formación de costra superficial de un oxisol de sabana en los llanos orientales de Colombia: caracterización micromorfológica en superficie. Centro Internacional de Agricultura Tropical (CIAT) Cali. 2008.

10. Gutiérrez B, Fierro L. Diagnóstico y diseño participativo en sistemas agroforestales, CORPOICA, Tibaitatá. 2006.

11. Harvey C. A., Villanueva C., Villacís J., Chacón M., Muñoz D, López, M, Ibrahim M, Gómez R, Taylor R, Martínez J, Navas A, Sáenz J, Sánchez D, Medina A, Vílchez $S$. Contribución de las cercas vivas a la productividad e integridad ecológica de los paisajes agrícolas en América Central. Agroforestería de las Américas. 2003.

12. ICA, Epidemiología Veterinaria, Censo bovinos Colombia. 2008.

13. INSTITUTO ALEXANDER VON HUMBOLDT - UNILLANOS. Plan de Acción regional en Biodiversidad para La Orinoquia. Diagnóstico final del eje 
"Conocer". Instituto "Alexander von Humboldt" - Universidad de Los Llanos. Informe inédito. 2003.

14. INSTITUTO ALEXANDER VON HUMBOLDT. Mapa de cuencas hidrográficas. http://www.humboldt.org.co/humboldt/mostrarpagina. php?codpage $=1111 \& u r l=$ http://orinoquia.humboldt.org.co. 2004

15. INSTITUTO GEOGRÁFICO AGUSTÍN CODAZZI, Corporación Colombiana de Investigación Agropecuaria, Zonificación de los conflictos de uso de las tierras en Colombia, capítulo II cobertura y uso actual de las tierras de Colombia. 2002.

16. INSTITUTO GEOGRÁFICO AGUSTÍN CODAZZI. Monitoreo del recurso suelo. Mapas recuperados el 10 de marzo de 2010 en: http://www.igac.gov.co:8080/igac web/contenidos/detalle documento plus.jsp ?idDocumento=111. 2010.

17. Martínez A, García F, Álvarez De León M. Acacia mangium, especie arbórea de uso múltiple para la Orinoquia Colombiana. CORPOICA, Villavicencio. 2006.

18. Martínez A, García F. Sistemas agroforestales con caucho: alternativa sostenible para la Orinoquia colombiana. Oferta tecnológica Amazonía Orinoquía. Sistema de producción caucho Área de Investigación Agroforestería, CORPOICA. 2005.

19. Meza R. Importancia y prácticas de sistemas agroforestales, Instituto Nacional de Investigaciones Forestales, Agrícolas y Pecuarias, INIFAP. 2003

20. Montagnini F. Estrategias para la recuperación de ecosistemas degradados: experiencias en América Latina. IV Congreso Forestal Centroamericano. Montelimar, Nicaragua, 15-17 Noviembre, 2000.

21. Muñoz D. Conocimiento local sobre cobertura arbórea en sistemas de producción ganadera en dos localidades de Costa Rica. Tesis Mag. Sc. CATIE, Turrialba. 2004.

22. Murgueitio E, Cuartas C, Naranjo J. Ganadería del futuro: Investigación para el desarrollo. Fundación CIPAV. Cali, Colombia. Capítulo 1. 2008.

23. Navia, J F. 2000. Agroforestería/Asesoría científica: Adolfo Hernández; Jesán Gómez/Coordinación de la Producción de Documentos Originales: Vicente Zapata S., Ed. D./ Cali, Colombia. 182 p.

24. Ojeda PA, Restrepo J. Sistemas silvopastoriles, una opción para el manejo sustentable de la ganadería. Santiago de Cali, Valle del Cauca - Colombia. 2003.

25. Ospina A, miembro de la fundación Ecovivero, Evolución de los componentes agroforestales. 2004.

26. Ospina A. Agroforestería: aportes conceptuales, metodológicos y prácticos para el estudio agroforestal, Cali - Colombia. Acasoc. 2003.

27. Pinzón W. "Modelo para evaluar el impacto económico y social de una política de expansión en la producción y uso de biodiesel en Colombia." Tesis de Maestría. Departamento de Ingeniería Industrial, Universidad de los Andes, Bogotá. 2010

28. Rincón A, Ligarreto G, Garay E. Producción de forraje en los pastos brachiaria decumbens cv. amargo y brachiaria brizantha cv. toledo, sometidos a tres 
frecuencias y a dos intensidades de defoliación en condiciones del piedemonte llanero colombiano. CORPOICA, Centro de Investigación La Libertad. Universidad Nacional de Colombia, Sede Bogotá. Facultad de Agronomía. 2008.

29. Rippstein G, Escobar G, Motta F. Agroecología y biodiversidad de las sabanas en los llanos orientales de Colombia. Centro Internacional de Agricultura Tropical, Cali - Colombia. 2001.

30. Rivas L., Hoyos P., Amézquita E., Molina D. L. Manejo y uso de los suelos de la Altillanura Colombiana - Análisis económico de una estrategia para su conservación y mejoramiento: Construcción de la capa arable. Proyecto de Evaluación de Impacto - Proyecto de Suelos. Convenio MADR-CIAT. Cali, Colombia. 2004.

31. Rubio D. Un nuevo escenario para modificar la distribución de la tierra en Colombia. Economía Colombiana 294: 90-95. 2002.

32. Sauceda M. Impacto del arreglo espacial del componente arbóreo en sistemas silvopastoriles sobre el nivel de sombreado y la conectividad estructural de los paisajes en los municipios de Belén y Matiguás, Nicaragua. Tesis Magister en Agroforestería Tropical. Centro Agronómico Tropical de Investigación y Enseñanza, Turrialba, Costa Rica. 2010.

33. Sotomayor A. Modelos agroforestales y desarrollo rural sustentable, Ingeniero Forestal MSc. Instituto forestal, Chile, 2004.

34. Vargas V, Sotomayor A. Modelos agroforestales: un sistema integrado en beneficio de la agricultura. Cartilla Agroforestal N. 1, Instituto Forestal, Santiago, Chile. 2004.

35. Viloria J. Geografía económica de la Orinoquia. Banco de la República. N 113. Documentos de trabajo sobre economía regional. 2009. 